\title{
Mapping of Cortical Activity in the First Two Decades of Life: A High-Density Sleep Electroencephalogram Study
}

\author{
Salomé Kurth, ${ }^{1}$ Maya Ringli, ${ }^{1}$ Anja Geiger, ${ }^{1,2}$ Monique LeBourgeois, ${ }^{3,4}$ Oskar G. Jenni, ${ }^{1,2}$ and Reto Huber ${ }^{1,2}$ \\ ${ }^{1}$ Child Development Center, Children's University Hospital Zurich, CH-8032 Zurich, Switzerland, ${ }^{2}$ Zurich Center for Integrative Human Physiology, \\ University of Zurich, CH-8057 Zurich, Switzerland, ${ }^{3}$ University of Colorado at Boulder, Department of Integrative Physiology, Boulder, Colorado 80309, \\ and ${ }^{4}$ The Warren Alpert Medical School of Brown University, Department of Psychiatry and Human Behavior, Providence, Rhode Island 02912
}

Evidence that electroencephalography (EEG) slow-wave activity (SWA) (EEG spectral power in the 1-4.5 Hz band) during non-rapid eye movement sleep (NREM) reflects plastic changes is increasing (Tononi and Cirelli, 2006). Regional assessment of gray matter development from neuroimaging studies reveals a posteroanterior trajectory of cortical maturation in the first three decades of life (Shaw et al., 2008). Our aim was to test whether this regional cortical maturation is reflected in regional changes of sleep SWA. We evaluated all-night high-density EEG (128 channels) in 55 healthy human subjects (2.4 -19.4 years) and assessed age-related changes in NREM sleep topography. As in adults, we observed frequency-specific topographical distributions of sleep EEG power in all subjects. However, from early childhood to late adolescence, the location on the scalp showing maximal SWA underwent a shift from posterior to anterior regions. This shift along the posteroanterior axis was only present in the SWA frequency range and remained stable across the night. Changes in the topography of SWA during sleep parallel neuroimaging study findings indicating cortical maturation starts early in posterior areas and spreads rostrally over the frontal cortex. Thus, SWA might reflect the underlying processes of cortical maturation. In the future, sleep SWA assessments may be used as a clinical tool to detect aberrations in cortical maturation.

\section{Introduction}

Evidence about the generation and functions of cortical activity during sleep is accumulating (Steriade, 2006; Tononi and Cirelli, 2006; Diekelmann and Born, 2010). In particular, the slow fluctuations of cortical activity during deep sleep have become a primary focus of many studies. First described by Steriade et al. (1993), these so-called "slow oscillations" are found in virtually all cortical neurons (Steriade et al., 2001; Timofeev et al., 2001). When slow oscillations are synchronized and involve the majority of cortical neurons in a certain region, they become visible in the surface electroencephalography (EEG) as slow waves (Vyazovskiy et al., 2009). The activity of sleep slow waves [slow wave activity (SWA), frequency range of $1-4.5 \mathrm{~Hz}$ ] reflects the depth of sleep (Borbély and Achermann, 2000) and seem also be related to processes of brain plasticity (Sejnowski and Destexhe, 2000; Steriade and Timofeev, 2003; Born et al., 2006; Tononi and Cirelli, 2006). Interestingly, sleep slow waves exhibit substantial changes from early childhood through adolescence (Jenni and Carskadon, 2004; Campbell and Feinberg, 2009), a developmental time window with massive changes in brain morphology and function (Johnson, 2001). The

Received April 12, 2010; revised July 8, 2010; accepted Aug. 9, 2010.

This work was supported by Swiss National Science Foundation Grant PP00A-114923 (R.H.), a research grant from the University Research Priority Program of the University of Zurich (R.H., 0.G.J.), National Institutes of Health Grant K01MH074643 (M.L.B.). A loan of computer hardware and software was provided by Electrical Geodesics. We thank the children and parents for their participation, Stephanie Cares, Margot Schaerer, and Jessica Bühler for technical assistance, and Giulio Tononi for the loan of technical material.

Correspondence should be addressed to Reto Huber, University Children's Hospital Zurich, Steinwiesstrasse 75, CH-8032 Zurich, Switzerland. E-mail: reto.huber@kispi.uzh.ch.

DOI:10.1523/JNEUROSCI.2532-10.2010

Copyright $\odot 2010$ the authors $\quad 0270-6474 / 10 / 3013211-09 \$ 15.00 / 0$ activity of slow waves during sleep increases in the first years of life, reaches its maximum shortly before puberty, and declines throughout adolescence (Feinberg, 1982; Gaudreau et al., 2001; Jenni et al., 2004; Campbell and Feinberg, 2009). This inverted U-shaped change of SWA as a function of subject age shows similarities with the time course of synapse density, which peaks between 4 and 6 years in prefrontal cortex (Huttenlocher and Dabholkar, 1997), and with cortical gray matter volume, as tracked by magnetic resonance imaging (MRI), presumably indirectly reflecting changes in synapse density (Giedd, 2004). Such longitudinal MRI studies indicate that not all cortical regions undergo maturational changes at the same speed and time (Giedd, 2004; Sowell et al., 2004; Shaw et al., 2008). Maturation follows a posteroanterior shift across the cortex (Giedd, 2004; Sowell et al., 2004; Shaw et al., 2008). According to these studies, occipital regions develop first and frontal areas mature last, which is consistent with the observation that many cognitive and behavioral functions related to the frontal cortex do not mature until late adolescence (Luna and Sweeney, 2004). Based on these observations, we examined whether the expression of SWA during sleep follows a similar spatial evolution during development.

Research supporting regional differences in the expression of sleep SWA stems from adults (Werth et al., 1996a; Cajochen et al., 1999; Finelli et al., 2001; Huber et al., 2004). These studies show that SWA is most pronounced over frontal cortices (Cajochen et al., 1999; Finelli et al., 2001). Furthermore, the origin of slow oscillations is particularly frequent over anterior regions (Massimini et al., 2004). Regional aspects of SWA are attributed to use dependent changes (Kattler et al., 1994) or, more specifically, to plastic changes induced by learning processes (Huber et al., 2004). Thus, 
the frontal predominance of SWA is in accordance with the observation that the frontal cortex is the brain area most "used" or plastic in adults (Horne, 1993; Couyoumdjian et al., 2010). Finally, the close relationship between cortical plasticity and sleep SWA may also be reflected by the association of slow waves and synaptic density or strength (Tononi and Cirelli, 2006). For example, using multiunit activity recordings in rats, Vyazovskiy et al. (2009) demonstrated a relationship between sleep homeostasis and the synchronization of neuronal population activity. Specifically, in early sleep, when sleep SWA is high, they found that most individual neurons stop or resume firing near synchrony; however, in late sleep, when SWA has dissipated, the entry into "on" and "off" periods was much more variable across neurons. Conversely, computer simulations electrophysiological and molecular data support a relationship between sleep homeostasis and synaptic strength, i.e., synaptic strength generally decreases during sleep and increases during wakefulness (Esser et al., 2007; Vyazovskiy et al., 2008). Together, it seems that synchronization depends on the level of synaptic strength: the denser and stronger synapses are, the faster they synchronize their activity and the larger is the resulting potential change measured by standard EEG over the cortex.

Thus, to explore the relationship between the extensive remodeling of brain circuits during cortical maturation and sleep EEG activity, we collected all-night high-density (HD) EEG recordings from 55 children and adolescents (age, 2.4-19.4 years). We found that the location on the scalp showing maximal SWA underwent a shift from posterior to anterior regions from early childhood to late adolescence. Because anatomical maturation starts in posterior areas and spreads rostrally over the frontal cortex, we conclude that SWA might reflect the underlying processes of cortical maturation.

\section{Materials and Methods}

Participants. A total of 55 healthy subjects (range, 2.4-19.4 years; 29 males) were recruited. Subjects underwent a telephone and questionnaire screening to exclude personal or family history of psychopathology, chronic diseases, sleep disorders, and current use of psychoactive agents or other medications. No participants traveled across more than one time zone in the 4 months before the study. Written informed consent was obtained from the parents or from the participants of full age after explanation of the study methods and aims. The procedures were approved by the local ethics committee, and the study was performed according to the Declaration of Helsinki.

Participants or parents completed daily sleep diaries. Participants also wore wrist actigraphs to ensure schedule compliance before the recording night. Subjects were required to refrain from alcohol and medication. No naps were allowed $24 \mathrm{~h}$ preceding the testing, but in children used to regular napping (i.e., nap opportunity each day and falling asleep at least $4 \mathrm{~d} /$ week), the subjects were permitted to nap on the day of assessment so as not to introduce heightened sleep pressure (this was the case in two children of ages 4.7 and 5.1 years). Even if changes in the level of sleep pressure in our young subjects existed, we believe that these did not affect the topographical power distribution for several reasons: (1) comparing baseline and sleep after sleep deprivation, Finelli et al. (2001) found a very similar topographical power distribution in adult subjects; and (2) we examined a later time window of sleep and found a similar EEG power distribution as in early sleep (see Results). Recordings in postpubertal females were scheduled to the follicular phase. This approach was chosen to prevent the variation of sleep EEG activity markers (e.g., spindle activity) as a function of the menstrual cycle phase (Driver et al., 1996).

EEG recording. All-night sleep EEG, electrooculogram, and electromyogram were recorded in 41 subjects (age range, 8.7-19.4 years; 23 males) in the sleep laboratory of the University Children's Hospital Zurich (Zurich, Switzerland). Of the 41 subjects, three pairs were siblings (six subjects). Fourteen children (age range, 2.4-8.0 years; six males) were recorded at home (in Providence, RI). Of this sample, one pair were siblings, and, twice, four children were siblings (two boys were monozygotic twins). In total, 55 subjects were recorded. We excluded two subjects from the analysis: one girl because the recording did not taking place during the appropriate menstrual phase, and one subject because bedtime reports identified the individual as an extremely short sleeper. Thus, 53 subjects were included in the analysis unless explicitly stated. All participants were monitored during 1 night using HD sleep EEG (Electrical Geodesics Sensor Net for long-term monitoring, 128 channels, referenced to a vertex electrode for direct visualization and to the average across all channels for data analysis; details follow). The nets were adjusted to the vertex, and the cap electrodes were filled with gel electrolyte. The use of gel ensured the maintenance of good signals even after $8-10 \mathrm{~h}$ (Landsness et al., 2009; Määttä et al., 2010). Impedances were measured after applying the gel and at the beginning of the recording. Electrode impedances were set below $50 \mathrm{k} \Omega$. In one subject, the electrodes were removed after $5 \mathrm{~h}$ because of discomfort. The sleep episode of each subject was scheduled according to individual reported bedtime. Subjects were awakened in the morning to allow school or job participation, resulting in variable bedtimes and rise times (variables not further examined).

Preprocessing. Data were sampled at $500 \mathrm{~Hz}(0.01-200 \mathrm{~Hz})$ and referenced to the vertex $(\mathrm{Cz})$. Then the EEG was bandpass filtered $(0.5-50 \mathrm{~Hz})$ and downsampled to $128 \mathrm{~Hz}$. In one subject with low-frequency sweating artifacts, a $0.75 \mathrm{~Hz}$ high-pass filter was used. Artifacts were rejected on a $20 \mathrm{~s}$ basis after visual inspection and if power exceeded a threshold based on a mean power value in the $0.75-4.5$ and $20-30 \mathrm{~Hz}$ bands (Huber et al., 2000). Poor quality EEG channels were excluded (on average, four channels per subject). In total, $11.1 \%$ of the epochs were rejected (no significant age-related differences; two subjects were excluded from this age comparison because of poor signal during $>1 \mathrm{~h}$ ). All additional analyses are based on re-referenced data: for every EEG sample, the value of each channel was divided by the average value across all 109 channels above the ears that were not excluded (only good quality channels).

The EEG was visually scored for sleep stages (20 s epochs, C3A2 or C4A1) based on American Academy of Sleep Medicine standard criteria (Iber et al., 2007). Non-rapid eye movement (NREM) sleep episodes were defined according to standard criteria (Rechtschaffen and Kales, 1968; Feinberg and Floyd, 1979) and adapted because of frequently occurring "skipped" rapid eye movement (REM) sleep after the first NREM sleep episode ("skipped" REM occurred in $26 \%$ of the nights). Specifically, similar to the studies of Jenni and Carskadon (2004) and Kurth et al. (2010), we manually subdivided the first NREM sleep episode if (1) the duration of the first NREM episode exceeded $120 \mathrm{~min}$, and (2) stage 3 sleep in the first NREM episode was interrupted for at least 12 continuous minutes of stage 1 sleep, stage 2 sleep, wakefulness, or movement time. If both criteria were met, the first NREM sleep episode was subdivided at the lowest SWA. If the criteria were not met but the hypnogram and the SWA time course appeared as skipped REM (i.e., apparent interruption of sleep stage 3, obvious drop in SWA), we subdivided the cycle manually ( $11 \%$ of the recordings). In one subject, only the first $7 \mathrm{~h}$ of data were included in the analysis because of poor signals. In another subject, the EEG signal was affected by artifacts in the frequency bands 14.5-15.5 and $22-23 \mathrm{~Hz}$ (bands omitted for the analysis).

Spectral analysis. For qualitative exploration, spectral analysis was performed for all channels [fast Fourier transform routine, Hanning window, $20 \mathrm{~s}$ epochs (averages of five 4-s epochs), frequency resolution of $0.25 \mathrm{~Hz}$ ]. The $20 \mathrm{~s}$ spectral power values were then averaged for a certain time window. Comparisons in EEG power spectra were assessed by ANOVA (one-way) with factor age group. When the ANOVA reached significance, we performed post hoc Scheffé's test for multiple comparisons (significance at the $5 \%$ level). The lowest frequency bins $(<1 \mathrm{~Hz})$ were excluded from the analysis because of possible interactions with the high-pass filter at $0.5 \mathrm{~Hz}$ (see above, Preprocessing). Spectral data was analyzed up to $25 \mathrm{~Hz}$. Based on the spectral profile (see Fig. 2), subsequent analyses were restricted to specific (commonly used) frequency bands, including slow waves $(1-4.5 \mathrm{~Hz})$, theta $(4.75-7.75 \mathrm{~Hz})$, alpha $(8-9.75 \mathrm{~Hz})$, sigma $(10-15 \mathrm{~Hz})$, and beta $(20-25 \mathrm{~Hz})$.

Power analysis and statistics. For additional investigation, power maps were calculated for the defined frequency bands for all channels. EEG 
Table 1. Visually scored sleep variables

\begin{tabular}{|c|c|c|c|c|c|c|}
\hline Age groups $(n=51)$ & $2-5$ years $(n=8)$ & $5-8$ years $(n=5)$ & $8-11$ years $(n=12)$ & $11-14$ years $(n=9)$ & $14-17$ years $(n=12)$ & $17-20$ years $(n=5)$ \\
\hline \multicolumn{7}{|l|}{ Sleep variables } \\
\hline Time in bed (min) & $651.6 \pm 18.4$ & $579.9 \pm 17.4$ & $528.0 \pm 9.9$ & $523.6 \pm 13.4$ & $469.6 \pm 13.8$ & $399.6 \pm 24.8$ \\
\hline Total sleep time (min) & $581.9 \pm 10.1$ & $520.6 \pm 16.7$ & $444.5 \pm 12.3$ & $469.5 \pm 17.1$ & $429.2 \pm 17.7$ & $363.5 \pm 15.1$ \\
\hline Sleep efficiency (\%) & $89.6 \pm 2.0$ & $89.8 \pm 1.5$ & $84.4 \pm 2.7$ & $89.7 \pm 2.4$ & $91.1 \pm 1.8$ & $91.4 \pm 2.1$ \\
\hline Sleep latency (min) & $24.8 \pm 7.3$ & $18.5 \pm 4.3$ & $24.8 \pm 2.5$ & $22.7 \pm 5.4$ & $20.6 \pm 3.6$ & $13.6 \pm 3.2$ \\
\hline Waking after sleep onset (min) & $46.5 \pm 14.2$ & $43.0 \pm 9.9$ & $62.1 \pm 13.8$ & $35.4 \pm 8.6$ & $23.5 \pm 5.0$ & $25.7 \pm 9.0$ \\
\hline Stage $1(\%)$ & $4.2 \pm 0.3$ & $3.5 \pm 0.8$ & $7.3 \pm 0.9$ & $7.5 \pm 1.0$ & $8.6 \pm 1.3$ & $5.4 \pm 1.6$ \\
\hline Stage $2(\%)$ & $47.8 \pm 2.2$ & $51.7 \pm 2.2$ & $46.5 \pm 2.4$ & $46.4 \pm 2.4$ & $52.4 \pm 1.3$ & $57.6 \pm 2.1$ \\
\hline SWS (\%) & $20.4 \pm 1.2$ & $20.0 \pm 1.0$ & $27.3 \pm 2.9$ & $26.4 \pm 2.2$ & $20.7 \pm 1.3$ & $17.8 \pm 3.4$ \\
\hline REM sleep (\%) & $27.6 \pm 1.3^{a}$ & $24.8 \pm 1.1$ & $18.8 \pm 1.2^{b}$ & $19.7 \pm 1.1^{b}$ & $18.3 \pm 1.4^{b}$ & $19.2 \pm 1.4^{b}$ \\
\hline Sleep cycle duration (min) & $89.8 \pm 3.4$ & $87.0 \pm 3.0$ & $94.2 \pm 5.3$ & $92.7 \pm 3.9$ & $96.6 \pm 3.5$ & $95.4 \pm 3.4$ \\
\hline
\end{tabular}

Sleep variables (mean \pm SE) of all subjects with at least $4.5 \mathrm{~h}$ of recorded sleep time and four sleep cycles subdivided into age groups (2.4-19.4 years, $n=51$ ). Sleep efficiency, Total sleep time expressed as a percentage of time in bed; Sleep latency, latency to the first occurrence of stage 2 sleep; Waking after sleep onset, expressed in minutes; sleep stages are expressed as a percentage of total sleep time; SWS, slow-wave sleep as stage 3 sleep; REM sleep, rapid eye movement sleep. Sleep cycle duration, includes only the first four sleep cycles. Because subjects were awoken in the morning, the last sleep cycle could not always be completed. Thus, we applied two additional criteria for the analysis of cycle duration: in subjects with only four cycles but with a missing REM sleep episode in the last cycle, the last cycle was excluded from the comparison $(n=6)$. Moreover, only sleep cycles containing a minimum of 75 epochs of NREM sleep were included. Sleep variables Time in bed and Total sleep time were excluded from group comparisons because sleep was restricted in the morning (for details, see Materials and Methods). A one-way ANOVA with factor age group was significant for stage 1 and stage 2 sleep, SWS, and REM sleep (all $p<0.05, F=0.6-7.7, \mathrm{df}=5$ ). Post hoc Scheffé tests revealed a significant group difference for REM sleep as indicated by letters (a,b $p<0.05$ ). Age groups with different letters differ significantly from each other.

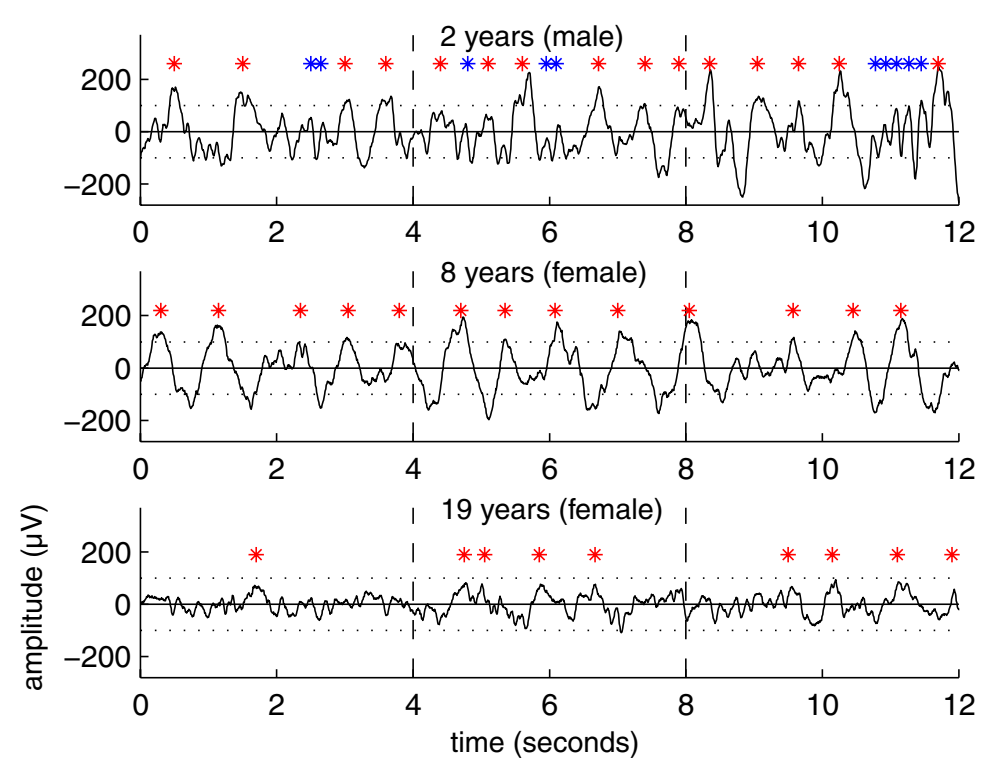

Figure 1. Tracings of slow-wave sleep for different ages. Twelve second EEG segments of slow-wave sleep in the first sleep cycle in three subjects of different ages (C3A2 derivation). Red stars indicate slow waves, and blue stars theta waves.

power for each electrode within a map was normalized to the average across the map. To further assess the age-related changes in topography along the posteroanterior axis, we compared the location of maximal power across five selected clusters of electrodes (see Fig. 4) (for more details, see supplemental Fig. S3, available at www.jneurosci.org as supplemental material). For each subject, the electrode with maximal power across all electrodes included in the five clusters was identified. The number of the cluster (from 1 to 5 ) that contained this electrode defined the region index (RI) (from 1 to 5). Thus, in each subject, the region index reflected the approximate location of maximal power for a defined frequency band. Next, individual region indices were averaged for each age group and statistically compared with ANOVA [two-way, factors age group (see Results) and time (early and late sleep; for details, see Fig. 2)], followed by Scheffe's multiple comparison test (significance at the 5\% level). To account for differences in sleep episode durations, we included the first 60 min of NREM sleep stages 2 and 3 (if no additional information is given in the text). Data variability is described as SEs. All analyses were performed with the software package MATLAB (MathWorks).

Anatomical localization of electrodes was verified in 35 subjects (8.719.4 years) using magnetic MRI and the positioning system Northern Digital Navigation System. Electrodes were digitized and coregistered with the subject's MRI using SofTaxic Optic (EMS Inc.) and the three- dimensional optical digitizer (Polaris Vicra; Northern Digital). T1-weighted anatomical images were obtained on a $3 \mathrm{~T}$ scanner, a General Electric Signa HDx. MR scans were collected in the axial plane (repetition time, 8.928 $\mathrm{ms}$; echo time, $3.496 \mathrm{~ms}$; flip angle, $13^{\circ}$; final resolution, $0.94 \times 0.94 \times 1.2 \mathrm{~mm})$.

\section{Results}

For the assessment of age-dependent changes, we subdivided the study population into the following six age groups: preschoolers (2-5 years), school-age children (5-8, 8-11, and 11-14 years), young adolescents (14-17 years), and older adolescents (17-20 years). First, we examined visually scored sleep variables to evaluate the sleep quality of the sample (Table 1). Sleep quality was good, i.e., rather short sleep latency and high sleep efficiency, and we did not find a difference between home and laboratory recordings for sleep latency and efficiency (unpaired $t$ tests, $p>$ 0.05 ). Sleep efficiency (group means from 84 to $91 \%$ ) and sleep latency (ranging from 13 to $25 \mathrm{~min}$ ) were consistent with laboratory-based measures reported from other studies (Mason et al., 2008). We did not observe any age effect on sleep latency and efficiency, which is in agreement with previous findings (Ohayon et al., 2004). However, we found age-dependent changes for REM sleep: in line with previous findings (Roffwarg et al., 1966), increasing age was associated with a significant decline in REM sleep (2-5 year olds, $28 \%$; groups including $8-20$ year olds, $18-20 \%$ ) (for details, see Table 1). In summary, we found minor age-related changes in sleep architecture based on standard visual scoring. Subsequent quantitative sleep EEG analysis was performed to investigate the predominant EEG activities during sleep.

\section{Age-related changes in the spectral profile of the sleep EEG}

Visual inspection of the sleep EEG revealed distinct age-related aspects. As expected, the three individual representative examples of different ages showed prominent slow waves (red marks) during deep sleep, but their expression was variable (Fig. 1). Most pronounced slow waves were found in school-age children (Fig. 


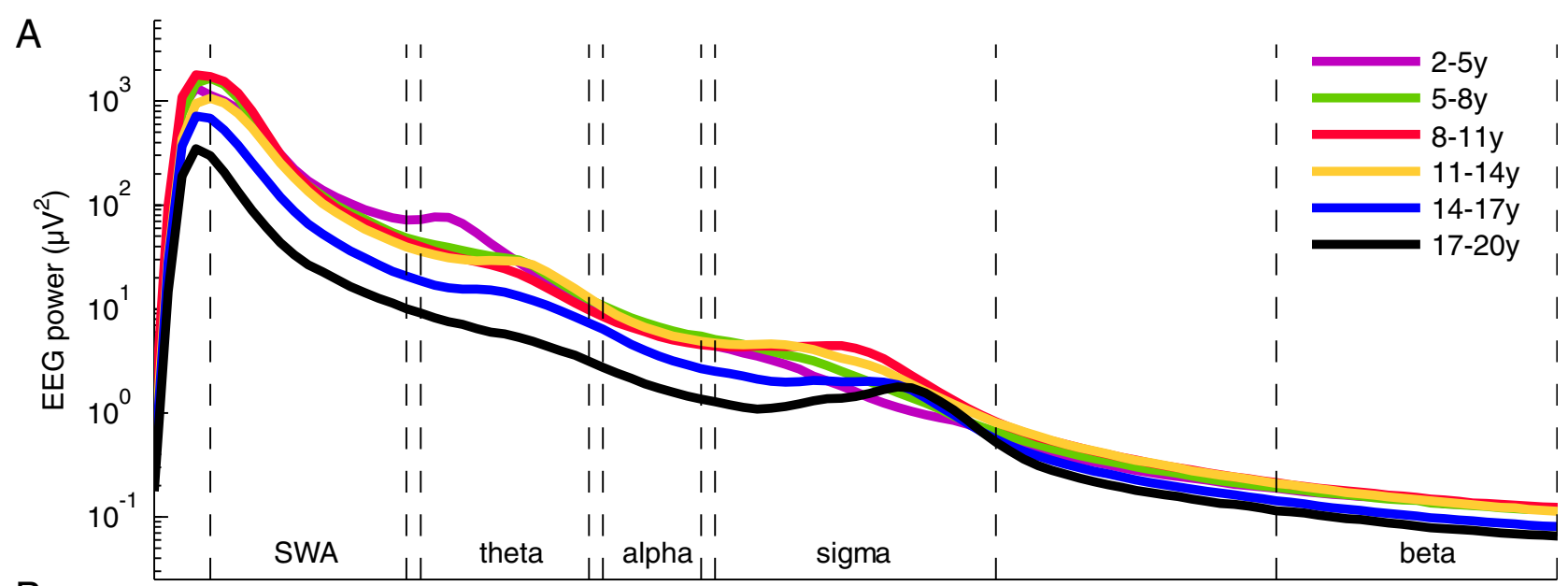

B

2-5y

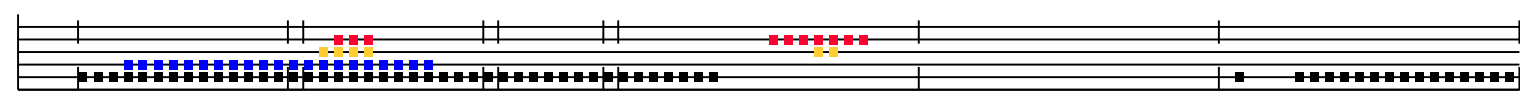

5-8y

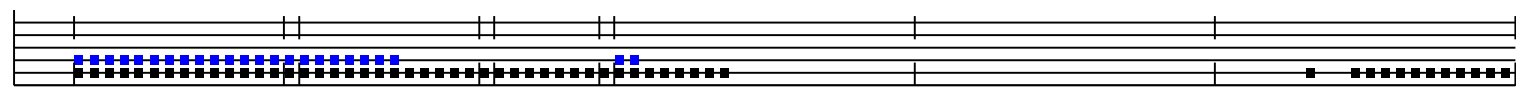

8-11y

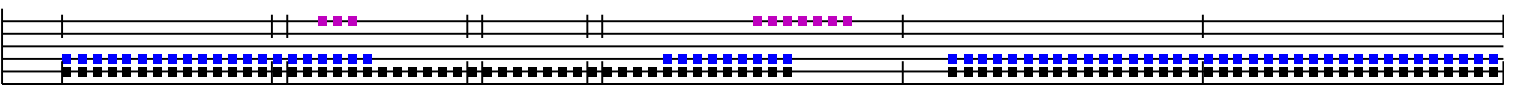

\section{1-14y}

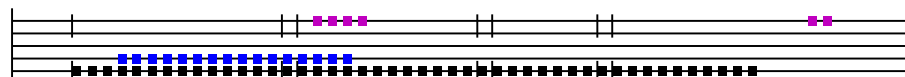

14-17y
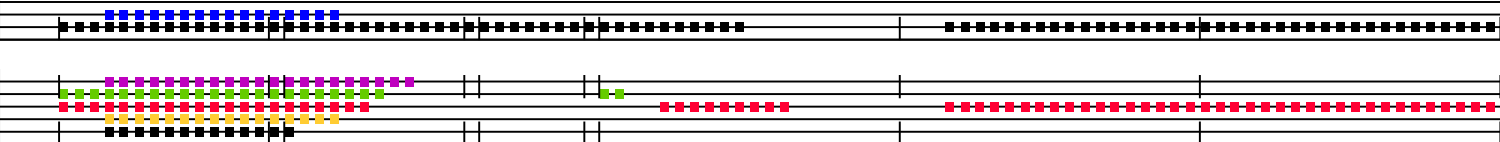

17-20y

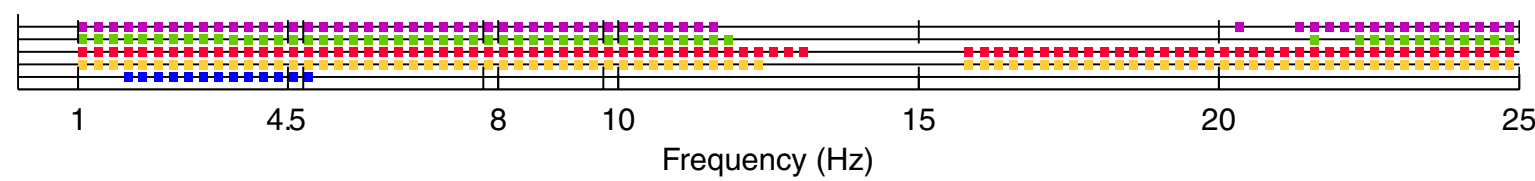

Figure 2. A, EEG power spectra during early NREM sleep. Average of all electrodes of the first 60 min NREM sleep stages 2 and 3 in six age groups (purple, $2-5$ years; green, $5-8$ years; red, $8-11$ years; yellow, 11-14 years; blue, $14-17$ years; black, $17-20$ years; $n=53$ ). Significant ANOVAs (see Materials and Methods) $(F=2.5-25.7$, df $=5$ ) were followed by groupwise comparisons as indicated in $\boldsymbol{B} . \boldsymbol{B}$, Posthoc Scheffés testing calculated for each $0.25 \mathrm{~Hz}$ bin and significant group differences indicated as dots $(p<0.05)$. Comparisons are color coded. Groups labeled on the ordinate were compared with color-coded groups for each particular frequency bin. A later time window of sleep showed a similar pattern of age group differences (supplemental data, available at www.jneurosci.org as supplemental material).

1, middle). The sleep EEG of the preschooler showed characteristic theta oscillations (Fig. 1, top, blue marks).

Next, we quantified age-related changes in the sleep EEG by spectral analysis and observed the classical spectral profile of the NREM sleep EEG (Fig. 2A). All age groups expressed the greatest power in the SWA range $(1-4.5 \mathrm{~Hz})$. A second and third local maximum was found in the theta $(4.75-7.75 \mathrm{~Hz})$ and sigma $(10-15 \mathrm{~Hz})$ frequency ranges of the power distribution. In general, the most pronounced group differences in the first $60 \mathrm{~min}$ of NREM sleep were present in the low frequencies (SWA and theta) (Fig. 2B). Fewer group differences were found in the alpha frequency range, whereas in the high sigma range, no differences between age groups were observed. Differences in the beta frequency range were similar to those observed in the alpha frequency band. We performed the same analysis for a later time window during the sleep period and found a similar pattern of age group differences (supplemental Fig. S1, available at www. jneurosci.org as supplemental material). We limited subsequent analyses to specific frequency ranges (SWA, theta, alpha, sigma, and beta) as defined by the spectral profile across all age groups (Fig. 2A).
Sleep EEG topography from early childhood through late adolescence

We next examined the topographical distribution of EEG power of the selected frequency ranges in the defined age groups (Fig. 3). All frequency ranges showed specific distribution patterns with maximal and minimal power expressions. These maxima and minima are indicated next to each plot and illustrate the overall time course of EEG power across age. In agreement with previous findings (Campbell and Feinberg, 2009), we found a significant power loss with an onset at approximately age 10 years for all frequency ranges. Only the slow-wave and sigma frequency ranges showed an increasing trend within the first decade of life.

Given the overall time course of EEG power across ages, we normalized each map to the average value across the map to assess age-related changes in topography. We found marked changes in the SWA range: in the two youngest groups, SWA was prevalent over the occipital lobe, whereas power showed a forward shift in the subsequent age groups. In contrast, other frequency ranges revealed only minor changes across age. Sigma power showed a prefrontal maximum that elongated toward central and occipital regions in the oldest group. A subdivision of the 


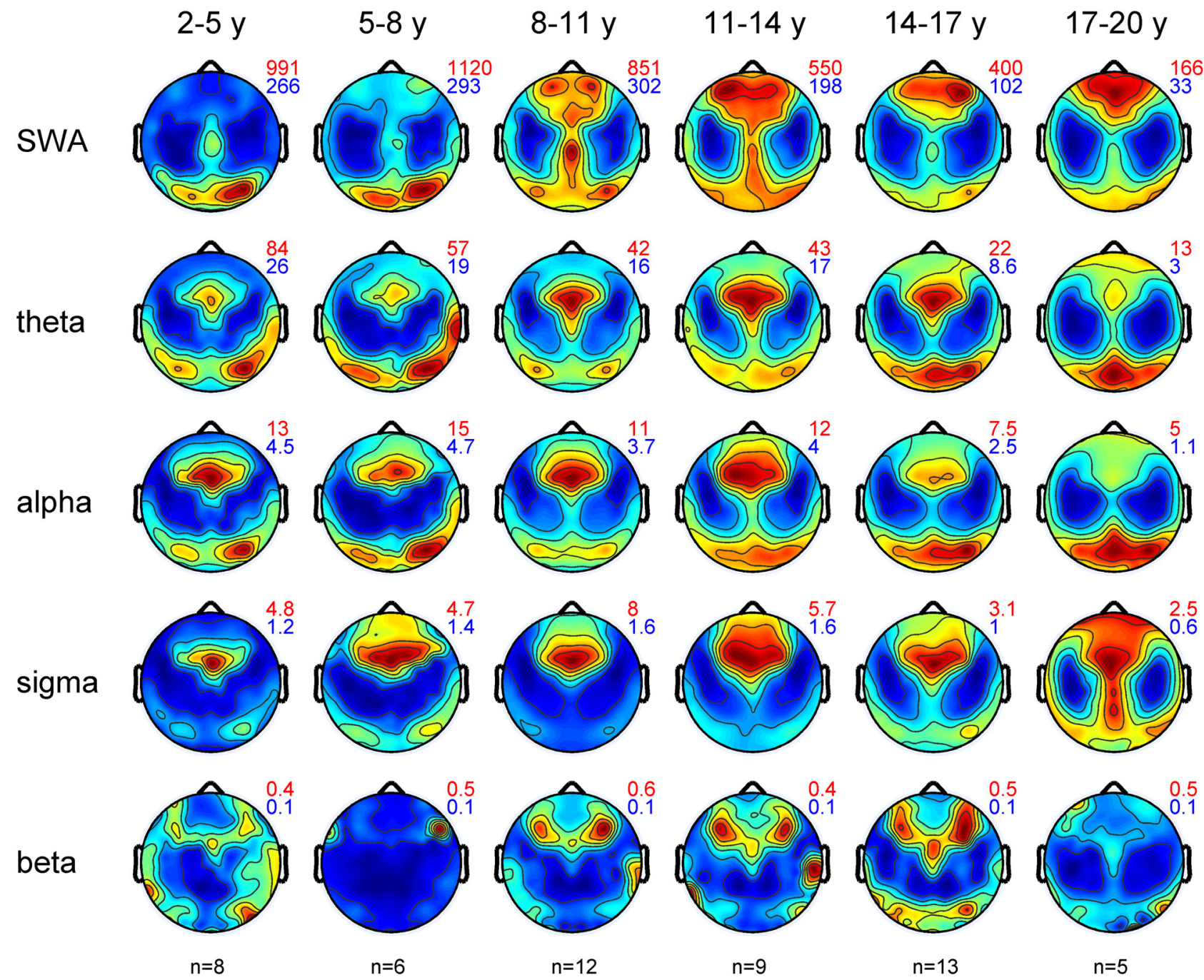

Minimal Power

Maximal Power

Figure 3. Maps of EEG power during NREM sleep. Topographical distribution of NREM sleep EEG power for the defined age groups and frequency ranges $(n=53)$. Maps are based on 109 derivations from the first $60 \mathrm{~min}$ of NREM sleep stages 2 and 3. Maps were normalized for each individual and then averaged for each age group. Values are color coded (maxima in red, minima in blue) and plotted on the planar projection of the hemispheric scalp model. To optimize contrast, each map was proportionally scaled, and values between the electrodes were interpolated. At the top right of the maps, numbers indicate maxima and minima (in square microvolts) for each plot.

sigma range into slow and fast spindles (Werth et al., 1997b) revealed that the latter accounted for the central relative increase, whereas slow spindles extended more toward frontal and occipital regions in the oldest group (data not shown). To quantify the variability of the topography across subjects, we calculated the relative error of the mean for each electrode (supplemental Fig. S2, available at www.jneurosci.org as supplemental material). The variability across subjects was low (with the exception of few electrodes). Variability was largest in the beta frequency range, indicating that these topographical distributions should be interpreted with caution. We also assessed the stability of topographical patterns across the night. The late sleep time window showed similar topographical distribution of EEG power in all frequency ranges and age groups (data not shown).

\section{Maximal expression of sleep EEG power along the} posteroanterior axis

For a statistical comparison of the shift of EEG power along the posteroanterior axis, we limited the analysis to five regions of interest (Fig. 4). A number ranging from 1 (most posterior) to 5 (most anterior) indicated the region of interest in which the power maxima for a certain frequency range and age group occurred (region index) (for more details, see Materials and Methods). We found significant age-dependent effects only for the SWA range. The RI of SWA showed a significant increase with age reflecting the shift of the power maxima from posterior to anterior cortical regions (from $1.3 \pm 0.2$ in the youngest to $4.0 \pm 0.8$ in the oldest group), with the most apparent change below the age of 11 years (Fig. 4). Other frequency ranges did not exhibit significant age-related changes of RI. We again tested this observation for stability across the night and found the similar age-related posteroanterior shift of SWA maxima in late sleep (and no age-related changes in other frequency ranges) (data not shown).

For an anatomical localization of the RI, we coregistered the electrodes with individual T1-weighted magnetic resonance images (for more details, see Materials and Methods). An orthogonal projection of the electrode onto the cortex showed the SWA maxima in preschoolers over the occipital lobe (lingual gyrus) 
and in older adolescents over the frontal lobe (medial frontal gyrus) (Table 2).

Figure 5 illustrates the ratio of EEG power of frontal $(\mathrm{F})$ to occipital $(\mathrm{O})$ electrodes and quantifies the age-related shift of maximal expression of SWA along the posteroanterior axis. The $\mathrm{F} / \mathrm{O}$ ratio of SWA correlated strongly with age $\left(R^{2}=\right.$ 0.67; $p<0.0001$ ) and remained stable across the night $\left(R^{2}=0.66\right.$ for late sleep; $p<0.0001$ ).

\section{Discussion}

This study examined sleep EEG topography from early childhood through adolescence. The main finding shows that the location with maximal SWA undergoes a shift from posterior to anterior regions across childhood and adolescence. None of the other frequency ranges demonstrated similar age-related spatial changes. This finding fundamentally expands previous knowledge about the maturation of the sleep EEG, because no comprehensive examination exists so far regarding (1) the first two decades of life and (2) the spatial resolution that allows for the description of regional differences. According to ana-

tomical (Von Economo, 1929), neuroimaging [e.g., MRI gray matter thickness (Shaw et al., 2008)], and behavioral [e.g., cognitive functions (Luna and Sweeney, 2004)] studies, cortical maturation follows a similar time course along the posteroanterior axis. The parallel time course of cortical maturation and sleep SWA may suggest that SWA reflects cortical plasticity during development.

Sleep depth exhibits prominent changes in the first two decades of life (Jenni and Carskadon, 2004; Feinberg and Campbell, 2010), and such changes in sleep depth are best characterized by EEG SWA during NREM sleep. Longitudinal and cross-sectional studies show an inverted U-shaped time course of SWA, i.e., an increase of SWA until puberty, followed by a decrease during adolescence (Feinberg, 1982; Gaudreau et al., 2001; Jenni et al., 2004; Campbell and Feinberg, 2009). Campbell and Feinberg alluded to the similarity of the time course of synapse density and SWA (Feinberg, 1982; Campbell and Feinberg, 2009), proposing that the decrease in SWA observed during adolescence reflects the decrease or pruning of synapses (Campbell and Feinberg, 2009; Feinberg and Campbell, 2010). Our data support this notion: we also observed the same overall decrease of SWA during adolescence. Recently, Vyazovskiy et al. (2009) used multiunit recordings in the rat and showed that increased synaptic strength allows for faster synchronization of cortical activity across neurons, resulting in larger-amplitude slow waves as observed with scalp EEG recordings. This observation provides a mechanistic explanation for the parallel time course of synapse density and overall SWA (Huttenlocher, 1979; Feinberg and Campbell, 2010; Kurth et al., 2010). Moreover, more and/or stronger synapses lead to increased energy consumption (Attwell and Laughlin, 2001). This finding might explain the similar time course of glucose usage, which shows a similar inverted U-shaped curve as SWA and synaptic density during the first two decades of life (Chugani et al., 1987). If synaptic strength is a key determinant for the level of synchronization in cortical networks, frequency ranges other
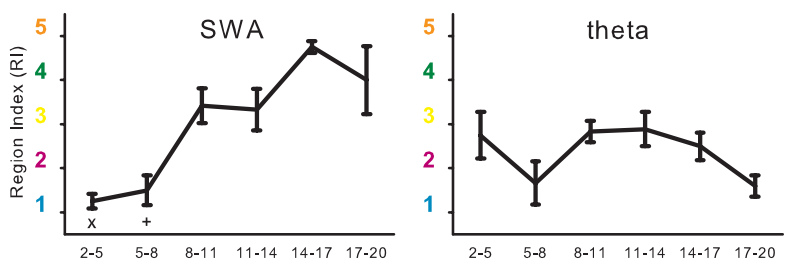

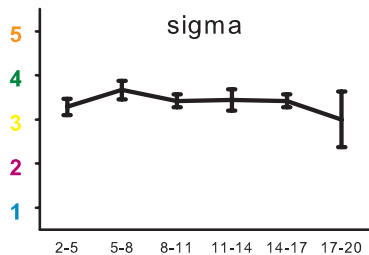

age groups (years)

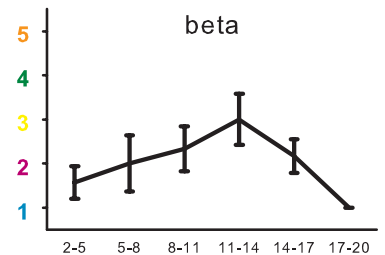

age groups (years)

Region index for selected frequency bands. Five cortical subregions along the inion-nasion axis are illustrated as (blue, RI1; purple, RI2; yellow, RI3; green, RI4; orange, RI5; the remaining electrodes are illustrated by with the subject's magnetic resonance images. Rls for age group are presented for the selected frequency bands. roup for the SWA range (Scheffé's tests, $p<0.05$; x indicates that $2-5$ years differ significantly from $8-11,11-14,14-17$, and 8 years differ significantly from $8-11,14-17$, and $17-20$ years).

than SWA should exhibit age-dependent changes. In fact, the decrease of sleep EEG power across adolescence is not limited to the SWA frequency range but also includes the theta range (Campbell and Feinberg, 2009). Again, we confirm this observation in our subject population. Finally, the effects of changes in the level of synchronization may not be limited to sleep. EEG recordings during wakefulness showed a similar inverted U-shaped time course of EEG power in the alpha frequency range (Gasser et al., 1988).

Previous sleep EEG studies during childhood and adolescence were restricted only to few derivations (Feinberg, 1982; Gaudreau et al., 2001; Jenni and Carskadon, 2004; Campbell and Feinberg, 2009). In contrast to former studies about the maturation of the sleep EEG using a limited number of electrodes, we successfully performed HD EEG recordings (with up to 128 electrodes) in children and adolescents to map changes in the sleep EEG with high spatial resolution. We believe high spatial resolution is important for two reasons. First, although sleep was considered a global phenomenon for many decades, a growing number of studies in recent years indicate otherwise, that is, sleep is a localized process. For example, several authors found a frontal predominance of SWA in adults (Werth et al., 1997a; Cajochen et al., 1999; Finelli et al., 2001; Huber et al., 2004). Second, anatomical, neuroimaging and behavioral studies report regional cortical maturation, which follows a tightly programmed course (Huttenlocher and Dabholkar, 1997; Luna and Sweeney, 2004; Shaw et al., 2008).

Similar to reports from adults, we observed regional differences in the distribution of EEG power for all classical frequency ranges. However, only the topography of EEG power in the SWA frequency range exhibited clear age-dependent changes. Thus, the most striking observation of our analysis was the substantial shift of the predominance of SWA during childhood and adolescence. Our anatomical localization of maximal occurrence of SWA revealed that, first, the maxima occurs over occipital lobe in 
Table 2. Anatomical localization of the electrodes along the anteroposterior axis

\begin{tabular}{|c|c|c|c|c|c|}
\hline \multirow[b]{2}{*}{ Electrode cluster [region index (1-5), F/0 index] } & \multicolumn{3}{|c|}{ Talairach coordinates (mean $\pm \mathrm{SE}$ ) } & \multirow{2}{*}{$\begin{array}{l}\text { Anatomical localization [lobe, gyrus, } \\
\text { Brodmann area }(B A)]\end{array}$} & \multirow[b]{2}{*}{ Range (mm) } \\
\hline & $x$ & $y$ & $Z$ & & \\
\hline 1 (most posterior) & $-3 \pm 2$ & $-92 \pm 3$ & $-14 \pm 3$ & Occipital lobe, lingual gyrus, BA 18 & 1 \\
\hline 1,0 & $-4 \pm 2$ & $-85 \pm 2$ & $33 \pm 3$ & Occipital lobe, cuneus, BA 19 & 0 \\
\hline 2 & $-4 \pm 2$ & $-62 \pm 2$ & $58 \pm 2$ & Parietal lobe, precuneus, BA 7 & 1 \\
\hline 2 & $-5 \pm 2$ & $-44 \pm 2$ & $65 \pm 2$ & Parietal lobe, postcentral gyrus, BA 5 & 0 \\
\hline 3 & $-4 \pm 2$ & $-9 \pm 3$ & $68 \pm 2$ & Frontal lobe, medial frontal gyrus, BA 6 & 1 \\
\hline 3 & $1 \pm 2$ & $9 \pm 2$ & $63 \pm 3$ & Frontal lobe, superior frontal gyrus, BA 6 & 4 \\
\hline 3 & $1 \pm 1$ & $41 \pm 2$ & $51 \pm 1$ & Frontal lobe, superior frontal gyrus, BA 8 & 3 \\
\hline $4, \mathrm{~F}$ & $2 \pm 1$ & $63 \pm 1$ & $24 \pm 2$ & Frontal lobe, medial frontal gyrus, BA 10 & 2 \\
\hline $5, F$ & $2 \pm 1$ & $67 \pm 1$ & $9 \pm 2$ & Frontal lobe, medial frontal gyrus, BA 10 & 2 \\
\hline $5, F$ & $3 \pm 1$ & $66 \pm 0$ & $-3 \pm 1$ & Frontal lobe, medial frontal gyrus, BA 10 & 1 \\
\hline 5 (most anterior) & $0 \pm 1$ & $56 \pm 1$ & $-17 \pm 1$ & Frontal lobe, medial frontal gyrus, BA 11 & 2 \\
\hline
\end{tabular}

Anatomical localization of the electrode clusters along the posteroanterior axis. Electrodes were digitized and coregistered with the subject's magnetic images. Electrodes of the RI (see Fig. 4) and F/0 index (see Fig. 5) are included. Average \pm SE Talairach coordinates are indicated for 35 subjects. The corresponding lobe, gyrus, and Brodmann area were detected using the Talairach Client (Lancaster et al., 1997, 2000). The range (in millimeters) refers to the distance of the nearest gray matter area with respect to the coordinates.

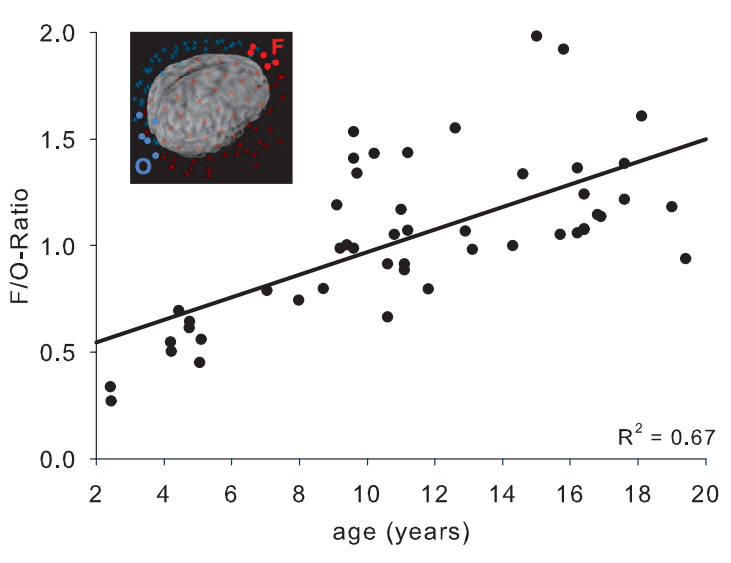

Figure 5. F/O ratio across age. SWA within a cluster of five electrodes in the frontal region (F) was averaged and divided by the value of five occipital electrodes ( 0 ). The cluster of electrodes included in the $\mathrm{F} / 0$ ratio is illustrated for an older adolescent (inset). The SWA F/0 ratio correlated significantly with age ( $p<0.001)$.

preschoolers, followed by parietal regions and posterior frontal lobe in school-age children (from 8 to 14 years) and, finally, the SWA maxima spreads to frontal lobes during adolescence. Young and older adolescents (after $\sim 14$ years of age) present a frontal predominance of SWA as found in adults (Fig. 3) (Werth et al., 1996b; Cajochen et al., 1999; Finelli et al., 2001). However, the frontal predominance of SWA in both younger and older adolescents is not yet as pronounced as in adults (Huber et al., 2004). These findings indicate that SWA topography undergoes maturational changes from childhood to late adolescence. The timing and the location of these changes in SWA topography are closely paralleled by anatomical and behavioral developmental changes. In the 1920s, Von Economo (1929) described cytoarchitonical changes spreading from back to front. Huttenlocher and Dabholkar (1997) also used postmortem samples to quantify synaptic densities of different cortical regions across age. According to their study, all areas showed an inverted U-shaped time course of synapse density. However, the age at which peak synaptic density was reached varied considerably: the occipital cortex was first and the frontal cortex was last. More recently, such regional changes in cortical maturation have been tracked by MRI. Several studies found that primary motor and sensory areas reach peak cortical thickness first, followed by secondary and association areas, and the frontal lobe matures last (Giedd, 2004; Sowell et al., 2004; Shaw et al., 2008). According to these imaging studies, cortical maturation starts in occipital poles and sensorimotor areas and spreads rostrally over the frontal cortex and caudally over parietal and then temporal cortex (Brecelj, 2003; Shaw et al., 2008). Finally, strong evidence for a local maturation of the cortex from behavioral observations exists. For example, executive functions, which are strongly dependent on frontal cortices (Tau and Peterson, 2010), are not fully mature until late adolescence (Spear, 2000; Luna and Sweeney, 2004). Conversely, visual acuity, a task predominantly performed by primary visual cortex (occipital lobe), matures in the first years of life (Teller, 1981).

In summary, SWA may not only reflect global changes in synapse density but also mirror the regional aspects of cortical maturation. A major factor underlying all such maturational changes is the age-dependent alteration of synaptic density. Changes in cortical volume and thickness across childhood and adolescence are attributed to the alterations in synaptic density and linked to the maturation of cognitive functions (Sowell et al., 2004). Changes in synaptic density lead to a change in the level of synchronization of the activity among neurons, which on the scalp is reflected by changes in the amplitude of rhythmic activities (Vyazovskiy et al., 2009). Although such a mechanism would explain the changes in SWA topography, we cannot exclude the possibility that additional factors may play a role in the relationship between cortical maturation and sleep SWA. For instance, a reduction in synapse number could be accompanied by a reduction in glial cells, resulting for example in changes of cortical thickness and volume (Paus, 2005). An association between glial cell activity and sleep SWA was recently proposed (Halassa et al., 2009).

Whatever factors during cortical maturation are responsible for the significant topographical changes of SWA, our results suggest that this relationship is frequency specific, because none of the other frequency ranges showed a similar spatial evolution of topography. This finding is in contrast to the global time course of EEG power across childhood and adolescence reported for several frequency ranges (Figs. 2, 3) (Campbell and Feinberg, 2009). Recent hypotheses about the function of sleep include sleep slow waves as the key electrophysiological feature during NREM sleep (Tononi and Cirelli, 2006; Diekelmann and Born, 2010). One of these hypotheses, the synaptic homeostasis hypothesis by Tononi and Cirelli (2006), directly relates sleep slow waves to synaptic plasticity. The hypothesis proposes that the activity of sleep slow waves directly reflects synaptic strength. Thus, the more a cortical network undergoes synaptic potentiation attributable to learning processes during wakefulness (Whitlock et al., 2006), the more SWA is expressed during subsequent sleep. The 
synaptic homeostasis hypothesis is supported by both electrophysiological and molecular data (Tononi and Cirelli, 2006). For instance, a major molecular marker of cortical plasticity, brain-derived neurotrophic factor (BDNF), is causally related to SWA (Faraguna et al., 2008). Blockage of BDNF by direct infusion of anti-BDNF results in a subsequent reduction of SWA. In contrast, local infusion of BDNF results in a corresponding increase of SWA. Also, experiments in humans support the relationship between cortical plasticity and sleep SWA. For example, Huber et al. $(2004,2008)$ reported the induction of plastic changes either during a learning task or directly by transcranial magnetic stimulation led to a local increase of SWA during subsequent sleep.

The current results further support a close relationship between cortical plasticity and sleep slow waves and suggest that SWA may be used to follow cortical maturation during sensitive periods, such as adolescence (Paus et al., 2008). "Overpruning" during adolescence has been linked to schizophrenia, mood disorders (Saugstad, 1994), autism, and mental retardation (Tessier and Broadie, 2009). Thus, the monitoring of synaptic remodeling during childhood and adolescence may increase our understanding of the pathophysiology of such disorders, lead to early diagnosis, and guide potential therapies (Woo and Crowell, 2005). Although MRI allows for a relative easy, non-invasive method for tracking anatomical changes, the relationship between anatomy and function are not trivial. The literature suggesting that MRI parameters predict behavior is contradictory. For example, studies reveal experience-dependent alterations of gray matter in specific areas (Draganski et al., 2004; Boyke et al., 2008; Jäncke et al., 2009), but the physiological or cellular basis of these changes is unclear (Jäncke et al., 2009). Only a thorough test of many cognitive functions using functional MRI and extensive behavioral testing may allow tracking of cortical functioning during development. Electroencephalography, i.e., the recording of the electrical activity of the brain, may provide a more direct assessment of cortical functioning. In particular, the activity during deep sleep permits a unique opportunity to quantify cortical network properties because the network during this sleep stage is, compared with waking, not disturbed by active behavior and to a lesser amount responding to external stimuli. The cortical activity during deep sleep is dominated by sleep SWA, and recent studies indicate that SWA reflects not only synaptic density (number) but also synaptic strength (e.g., levels of AMPA receptors per synapse) and synaptic efficacy (magnitude of the physiological effects, e.g., postsynaptic currents) (Tononi and Cirelli, 2006). We propose that the regional expression of SWA across cortical maturation may be a promising marker for plastic changes during childhood and adolescence, thus making sleep SWA a powerful tool to investigate cortical maturation in health and disease.

\section{References}

Attwell D, Laughlin SB (2001) An energy budget for signaling in the grey matter of the brain. J Cereb Blood Flow Metab 21:1133-1145.

Borbély AA, Achermann P (2000) Homeostasis of human sleep and models of sleep regulation. In: Principles and practice of sleep medicine (Kryger MH, Roth T, Dement WC, eds), pp 377-390. Philadelphia: Saunders.

Born J, Rasch B, Gais S (2006) Sleep to remember. Neuroscientist 12:410-424.

Boyke J, Driemeyer J, Gaser C, Büchel C, May A (2008) Training-induced brain structure changes in the elderly. J Neurosci 28:7031-7035.

Brecelj J (2003) From immature to mature pattern ERG and VEP. Doc Ophthalmol 107:215-224.

Cajochen C, Foy R, Dijk DJ (1999) Frontal predominance of a relative increase in sleep delta and theta EEG activity after sleep loss in humans. Sleep Res Online 2:65-69.
Campbell IG, Feinberg I (2009) Longitudinal trajectories of non-rapid eye movement delta and theta EEG as indicators of adolescent brain maturation. Proc Natl Acad Sci U S A 106:5177-5180.

Chugani HT, Phelps ME, Mazziotta JC (1987) Positron emission tomography study of human brain functional development. Ann Neurol 22:487-497.

Couyoumdjian A, Sdoia S, Tempesta D, Curcio G, Rastellini E, De Gennaro L, Ferrara M (2010) The effects of sleep and sleep deprivation on taskswitching performance. J Sleep Res 19:64-70.

Diekelmann S, Born J (2010) The memory function of sleep. Nat Rev 11:114-126.

Draganski B, Gaser C, Busch V, Schuierer G, Bogdahn U, May A (2004) Neuroplasticity: changes in grey matter induced by training. Nature 427:311-312.

Driver HS, Dijk DJ, Werth E, Biedermann K, Borbély AA (1996) Sleep and the sleep electroencephalogram across the menstrual cycle in young healthy women. J Clin Endocrinol Metab 81:728-735.

Esser SK, Hill SL, Tononi G (2007) Sleep homeostasis and cortical synchronization. I. Modeling the effects of synaptic strength on sleep slow waves. Sleep 30:1617-1630.

Faraguna U, Vyazovskiy VV, Nelson AB, Tononi G, Cirelli C (2008) A causal role for brain-derived neurotrophic factor in the homeostatic regulation of sleep. J Neurosci 28:4088-4095.

Feinberg I (1982) Schizophrenia: caused by a fault in programmed synaptic elimination during adolescence? J Psychiatr Res 17:319-334.

Feinberg I, Campbell IG (2010) Sleep EEG changes during adolescence: an index of a fundamental brain reorganization. Brain Cogn 72:56-65.

Feinberg I, Floyd TC (1979) Systematic trends across the night in human sleep cycles. Psychophysiology 16:283-291.

Finelli LA, Borbély AA, Achermann P (2001) Functional topography of the human nonREM sleep electroencephalogram. Eur J Neurosci 13:2282-2290.

Gasser T, Verleger R, Bächer P, Sroka L (1988) Development of the EEG of school-age children and adolescents. I. Analysis of band power. Electroencephalogr Clin Neurophysiol 69:91-99.

Gaudreau H, Carrier J, Montplaisir J (2001) Age-related modifications of NREM sleep EEG: from childhood to middle age. J Sleep Res 10:165-172.

Giedd JN (2004) Structural magnetic resonance imaging of the adolescent brain. Ann NY Acad Sci 1021:77-85.

Halassa MM, Florian C, Fellin T, Munoz JR, Lee SY, Abel T, Haydon PG, Frank MG (2009) Astrocytic modulation of sleep homeostasis and cognitive consequences of sleep loss. Neuron 61:213-219.

Horne JA (1993) Human sleep, sleep loss and behaviour. Implications for the prefrontal cortex and psychiatric disorder. $\mathrm{Br} \mathrm{J}$ Psychiatry 162:413-419.

Huber R, Graf T, Cote KA, Wittmann L, Gallmann E, Matter D, Schuderer J, Kuster N, Borbély AA, Achermann P (2000) Exposure to pulsed highfrequency electromagnetic field during waking affects human sleep EEG. Neuroreport 11:3321-3325.

Huber R, Ghilardi MF, Massimini M, Tononi G (2004) Local sleep and learning. Nature 430:78-81.

Huber R, Määttä S, Esser SK, Sarasso S, Ferrarelli F, Watson A, Ferreri F, Peterson MJ, Tononi G (2008) Measures of cortical plasticity after transcranial paired associative stimulation predict changes in electroencephalogram slow-wave activity during subsequent sleep. J Neurosci 28:7911-7918.

Huttenlocher PR (1979) Synaptic density in human frontal cortex: developmental changes and effects of aging. Brain Res 163:195-205.

Huttenlocher PR, Dabholkar AS (1997) Regional differences in synaptogenesis in human cerebral cortex. J Comp Neurol 387:167-178.

Iber C, Ancoli-Israel S, Chesson AL, Quan SF, eds (2007) The AASM manual for the scoring of sleep and associated events: rules, terminology and technical specifications, Ed 1. Westchester, IL: American Academy of Sleep Medicine.

Jäncke L, Koeneke S, Hoppe A, Rominger C, Hänggi J (2009) The architecture of the golfer's brain. PLoS One 4:e4785.

Jenni OG, Carskadon MA (2004) Spectral analysis of the sleep electroencephalogram during adolescence. Sleep 27:774-783.

Jenni OG, Borbely AA, Achermann P (2004) Development of the nocturnal sleep electroencephalogram in human infants. Am J Physiol 286:R528-R538. 
Johnson MH (2001) Functional brain development in humans. Nat Rev 2:475-483.

Kattler H, Dijk DJ, Borbély AA (1994) Effect of unilateral somatosensory stimulation prior to sleep on the sleep EEG in humans. J Sleep Res 3:159-164.

Kurth S, Jenni OG, Riedner BA, Tononi G, Carskadon MA, Huber R (2010) Characteristics of sleep slow-waves in children and adolescents. Sleep 33:475-480.

Lancaster JL, Rainey LH, Summerlin JL, Freitas CS, Fox PT, Evans AC, Toga AW, Mazziotta JC (1997) Automated labeling of the human brain: a preliminary report on the development and evaluation of a forwardtransform method. Hum Brain Mapp 5:238-242.

Lancaster JL, Woldorff MG, Parsons LM, Liotti M, Freitas CS, Rainey L, Kochunov PV, Nickerson D, Mikiten SA, Fox PT (2000) Automated Talairach atlas labels for functional brain mapping. Hum Brain Mapp 10:120-131.

Landsness EC, Crupi D, Hulse BK, Peterson MJ, Huber R, Ansari H, Coen M, Cirelli C, Benca RM, Ghilardi MF, Tononi G (2009) Sleep-dependent improvement in visuomotor learning: a causal role for slow waves. Sleep 32:1273-1284.

Luna B, Sweeney JA (2004) The emergence of collaborative brain function: FMRI studies of the development of response inhibition. Ann NY Acad Sci 1021:296-309.

Määttä S, Landsness E, Sarasso S, Ferrarelli F, Ferreri F, Ghilardi MF, Tononi G (2010) The effects of morning training on night sleep: a behavioral and EEG study. Brain Res Bull 82:118-123.

Mason TB 2nd, Teoh L, Calabro K, Traylor J, Karamessinis L, Schultz B, Samuel J, Gallagher PR, Marcus CL (2008) Rapid eye movement latency in children and adolescents. Pediatr Neurol 39:162-169.

Massimini M, Huber R, Ferrarelli F, Hill S, Tononi G (2004) The sleep slow oscillation as a traveling wave. J Neurosci 24:6862-6870.

Ohayon MM, Carskadon MA, Guilleminault C, Vitiello MV (2004) Metaanalysis of quantitative sleep parameters from childhood to old age in healthy individuals: developing normative sleep values across the human lifespan. Sleep 27:1255-1273.

Paus T (2005) Mapping brain maturation and cognitive development during adolescence. Trends Cogn Sci 9:60-68.

Paus T, Keshavan M, Giedd JN (2008) Why do many psychiatric disorders emerge during adolescence? Nat Rev 9:947-957.

Rechtschaffen A, Kales A (1968) A manual of standardized terminology, techniques and scoring system for sleep stages of human subjects. Washington, DC: US Public Health Service, US Government Printing Office.

Roffwarg HP, Muzio JN, Dement WC (1966) Ontogenetic development of the human sleep-dream cycle. Science 152:604-619.

Saugstad LF (1994) The maturational theory of brain development and cerebral excitability in the multifactorially inherited manic-depressive psychosis and schizophrenia. Int J Psychophysiol 18:189-203; discussion 187-188.

Sejnowski TJ, Destexhe A (2000) Why do we sleep? Brain Res 886:208-223.

Shaw P, Kabani NJ, Lerch JP, Eckstrand K, Lenroot R, Gogtay N, Greenstein D, Clasen L, Evans A, Rapoport JL, Giedd JN, Wise SP (2008) Neurode- velopmental trajectories of the human cerebral cortex. J Neurosci 28:3586-3594.

Sowell ER, Thompson PM, Leonard CM, Welcome SE, Kan E, Toga AW (2004) Longitudinal mapping of cortical thickness and brain growth in normal children. J Neurosci 24:8223-8231.

Spear LP (2000) The adolescent brain and age-related behavioral manifestations. Neurosci Biobehav Rev 24:417-463.

Steriade M (2006) Grouping of brain rhythms in corticothalamic systems. Neuroscience 137:1087-1106.

Steriade M, Timofeev I (2003) Neuronal plasticity in thalamocortical networks during sleep and waking oscillations. Neuron 37:563-576.

Steriade M, Contreras D, Curró Dossi R, Nuñez A (1993) The slow $(<1 \mathrm{~Hz})$ oscillation in reticular thalamic and thalamocortical neurons: scenario of sleep rhythm generation in interacting thalamic and neocortical networks. J Neurosci 13:3284-3299.

Steriade M, Timofeev I, Grenier F (2001) Natural waking and sleep states: a view from inside neocortical neurons. J Neurophysiol 85:1969-1985.

Tau GZ, Peterson BS (2010) Normal development of brain circuits. Neuropsychopharmacology 35:147-168.

Teller DY (1981) The development of visual-acuity in human and monkey infants. Trends Neurosci 4:21-24.

Tessier CR, Broadie K (2009) Activity-dependent modulation of neural circuit synaptic connectivity. Front Mol Neurosci 2:8.

Timofeev I, Grenier F, Steriade M (2001) Disfacilitation and active inhibition in the neocortex during the natural sleep-wake cycle: an intracellular study. Proc Natl Acad Sci U S A 98:1924-1929.

Tononi G, Cirelli C (2006) Sleep function and synaptic homeostasis. Sleep Med Rev 10:49-62.

Von Economo CV (1929) The cytoarchitectonics of the human cerebral cortex. London: Oxford Medical Publications.

Vyazovskiy VV, Cirelli C, Pfister-Genskow M, Faraguna U, Tononi G (2008) Molecular and electrophysiological evidence for net synaptic potentiation in wake and depression in sleep. Nat Neurosci 11:200-208.

Vyazovskiy VV, Olcese U, Lazimy YM, Faraguna U, Esser SK, Williams JC, Cirelli C, Tononi G (2009) Cortical firing and sleep homeostasis. Neuron 63:865-878.

Werth E, Achermann P, Borbély AA (1996a) Brain topography of the human sleep EEG: antero-posterior shifts of spectral power. Neuroreport 8:123-127.

Werth E, Achermann P, Dijk DJ, Borbely AA (1996b) Topographic distribution of spindle frequency activity in the sleep EEG. J Sleep Res 5 [Suppl 1]:252.

Werth E, Achermann P, Borbély AA (1997a) Fronto-occipital EEG power gradients in human sleep. J Sleep Res 6:102-112.

Werth E, Achermann P, Dijk DJ, Borbély AA (1997b) Spindle frequency activity in the sleep EEG: individual differences and topographic distribution. Electroencephalogr Clin Neurophysiol 103:535-542.

Whitlock JR, Heynen AJ, Shuler MG, Bear MF (2006) Learning induces long-term potentiation in the hippocampus. Science 313:1093-1097.

Woo TU, Crowell AL (2005) Targeting synapses and myelin in the prevention of schizophrenia. Schizophr Res 73:193-207. 